\title{
Laparoscopic Surgical Approach for the Treatment of Pelvic Piriformis Syndrome
}

\author{
Ahmet Kale ${ }^{1}$, Gulfem Basol ${ }^{1}$, Betul Kuru ${ }^{1}$, Elif Gundogdu ${ }^{1}$, Emre Mat ${ }^{1}$, Gazi Yildiz ${ }^{1}$, \\ Navdar Dogus Uzun ${ }^{2}$, and Taner Usta ${ }^{3}$ \\ ${ }^{1}$ Istanbul Dr Lufti Kirdar Kartal Egitim ve Arastirma Hastanesi \\ ${ }^{2}$ Mardin Midyat State Hispital \\ ${ }^{3}$ Acibadem Universitesi Tip Fakultesi
}

October 26, 2020

\begin{abstract}
Background: Piriformis syndrome is an uncommon disease result from compression of the sciatic nerve by the piriformis muscle. Symptoms may include pain and numbness in the buttocks and down the leg. This study analyzes the laparoscopic surgical treatments of pelvic piriformis syndrome. Presentation: We report two cases of pelvic piriformis syndrome diagnosed in our hospital. The first case was a 40 years old woman with a 7-year history of intermittent low back and sciatalgia on her right side. The severity of the pain was $10 / 10$ on the visual analog scale(VAS). Hyperesthesia and cutaneous allodynia were observed in the right sciatic nerve dermatome. The laparoscopic decompression surgery was performed to release the right sciatic nerve from the aberrant piriformis muscle. Postoperative 3 and 6 months follow-up, her VAS decreased to 0/10. The second case was a 30 years old woman with a 2-year history of sciatalgia on her left side. Her VAS was 9/10. The laparoscopic decompression surgery was performed to release the left sciatic nerve and left sacral nerve S2 dermatome from the aberrant piriformis muscle. Postoperative 3 and 6 months follow-up, her VAS decreased to 1/10. Conclusion: Due to the very few cases in the literature, pelvic piriformis syndrome is thought to be an exclusively clinical diagnosis. The sciatica is refractory despite appropriate conservative treatments; laparoscopic exploration of the pelvic nerves and piriformis muscle and decompression surgery can be a good option. Keywords: Laparoscopy, pain, piriformis muscle syndrome, sacral nerve root, the sciatic nerve.
\end{abstract}

\section{Laparoscopic Surgical Approach for the Treatment of Pelvic Piriformis Syndrome}

\section{Treatment of Pelvic Piriformis Syndrome}

Corresponding Author:

Betul Kuru, M.D.

Department of Obstetrics and Gynecology, University of Health Sciences Turkey Kartal Dr. Lutfi Kirdar Research and Training Hospital, Istanbul, Turkey

Postal address: D-100 Guney Yanyol, Cevizli Mevkii No:47, Kartal Dr. Lutfi Kirdar Research and Training Hospital, Department of Obstetrics and Gynecology, 34865, Kartal, Istanbul, Turkey.

E-mail: drbetulkuru@yahoo.com.tr

Phone number: +905063092999

Ahmet Kale, Prof Dr.

Department of Obstetrics and Gynecology, University of Health Sciences Turkey Kartal Dr. Lutfi Kirdar Research and Training Hospital, Istanbul, Turkey 
Gulfem Basol, M.D.

Department of Obstetrics and Gynecology, University of Health Sciences Turkey Kartal Dr. Lutfi Kirdar Research and Training Hospital, Istanbul, Turkey

Elif Cansu Gundogdu, M.D.

Department of Obstetrics and Gynecology, University of Health Sciences Turkey Kartal Dr. Lutfi Kirdar Research and Training Hospital, Istanbul, Turkey

Emre Mat, M.D.

Department of Obstetrics and Gynecology, University of Health Sciences Turkey Kartal Dr. Lutfi Kirdar Research and Training Hospital, Istanbul, Turkey

Gazi Yildiz, M.D.

Department of Obstetrics and Gynecology, University of Health Sciences Turkey Kartal Dr. Lutfi Kirdar Research and Training Hospital, Istanbul, Turkey

Navdar Dogus Uzun, M.D.

Department of Obstetrics and Gynecology, Mardin Midyat State Hospital, Mardin, Turkey

Taner Usta, Prof Dr.

Department of Obstetrics and Gynecology, University of Acibadem Hospital, Istanbul, Turkey

\section{Abstract}

\section{Background:}

Piriformis syndrome is an uncommon disease that results from compression of the sciatic nerve by the piriformis muscle. Symptoms may include pain and numbness in the buttocks and down the leg. This study analyzes the laparoscopic surgical treatments of pelvic piriformis syndrome.

Presentation: We report two cases of pelvic piriformis syndrome diagnosed in our hospital. The first case was a 40 years old woman with a 7-year history of intermittent low back and sciatalgia on her right side. The severity of the pain was 10/10 on the visual analog scale(VAS). Hyperesthesia and cutaneous allodynia were observed in the right sciatic nerve dermatome. The laparoscopic decompression surgery was performed to release the right sciatic nerve from the aberrant piriformis muscle. Postoperative 3 and 6 months follow-up, her VAS decreased to $0 / 10$. The second case was a 30 years old woman with a 2-year history of sciatalgia on her left side. Her VAS was $9 / 10$. The laparoscopic decompression surgery was performed to release the left sciatic nerve and left sacral nerve S2 dermatome from the aberrant piriformis muscle. Postoperative 3 and 6 months follow-up, her VAS decreased to 1/10.

Conclusion: Due to the very few cases in the literature, pelvic piriformis syndrome is thought to be an exclusively clinical diagnosis. The sciatica is refractory despite appropriate conservative treatments; laparoscopic exploration of the pelvic nerves and piriformis muscle and decompression surgery can be a good option.

Keywords: Laparoscopy, pain, piriformis muscle syndrome, sacral nerve root, sciatic nerve.

\section{INTRODUCTION}

Piriformis syndrome is a non-discogenic sciatalgia caused by the piriformis muscle's impingement of the sciatic nerve, inducing buttock pain and worsening pain with sitting. The piriformis is a flat and triangularshaped muscle originates on the anterior surface of the second through the fourth sacral vertebra, sacroiliac joint capsule, sacrotuberous ligament, and its tendon attaches medial aspect of the greater trochanter. Its principal function is to externally rotate and abduct the hip. It should be noted that resetting the hip's 
position changes the function of the piriformis muscle; with the hip in flexion, the piriformis is an abductor; however, with the hip in extension, it is an external rotator (1).

There are different anatomical structures around the piriformis muscle, and the most important one is the sciatic nerve. The piriformis muscle can also irritate the nearby sciatic nerve and cause pain, numbness, and tingling along the leg's back and into the foot, similar to sciatica pain. Various causes of entrapment of sciatic nerve due to piriformis muscle have been reported: congenital anomalies where the sciatic nerve or one of its branches passes through the piriformis muscle, gluteal trauma, posttraumatic scarring, and piriformis muscle hypertrophy (2).

Various specific tests stretch the piriformis can be used for the diagnosis of piriformis syndrome. Freiberg's test elicits pain by passively internal rotation of the extended hip when the patient is in a supine position. The FAIR test is done by flexing, adducting, and internally rotating the hip. The test is considered positive if stretching of the piriformis muscle produces pain. The Pace test is performed by resisting the hip's abduction and external rotation while the patient is in a seated position. The Beatty test is another diagnostic test for piriformis syndrome. In this test, the patient lies on the unaffected side, lifting and holding the superior knee approximately 4 inches off the examination table. If sciatic symptoms are recreated, the test result is positive (3).

There is no definitive diagnostic test. X-ray radiography, magnetic resonance imaging (MRI) and electrophysiological evaluation can be performed for the differential diagnosis. MRI of the lumbar spine and pelvis would be recommended to evaluate a discogenic cause for sciatalgia and rule out sciatic nerve compression by intrapelvic or extrapelvic lesions (4).

Sciatic nerve compression in the pelvic area with piriformis muscle causing sciatalgia is rare, and diagnosis is often difficult since there is no gold standard test for this condition. The imaging technique relating to the piriformis muscle made very little to help our understanding of piriformis muscle syndrome. In some cases, there are some signal abnormalities either from the piriformis muscle or the sciatic nerve itself. When extrapelvic etiological causes of piriformis muscle syndrome are excluded, intrapelvic sciatic nerve compression is highly suspected due to aberrant piriformis muscle. Laparoscopic intrapelvic sciatic nerve decompression should be considered a surgical treatment technique for pelvic piriformis muscle syndrome (5).

The first aim of our article was to create a new perspective of the aberrant piriformis muscle bundle as a seldom diagnosed cause of sciatalgia. The second aim was to present an alternative laparoscopic approach to transect the intrapelvic aberrant piriformis muscle to relieve sciatic nerve compression.

\section{History and Examination}

The first patient was a 40 years old woman with a 7-year history of intermittent low back and sciatica pain on her right side. The patient reported her symptoms like shooting, stabbing or burning sensation. The severity of the pain was $10 / 10$ on the visual analog scale. Her symptoms were continuous and particularly severe when prolonged sitting and standing position and became unbearable with physical activity. She had no sexual activity for two years because of severe dyspareunia. She could walk intermittently because of her leg pain. She had undergone a total abdominal hysterectomy to manage chronic pelvic pain previously, but surgical treatment failed. After the ultrasound-guided injection of local anesthetic into the piriformis muscle, medical, and physical treatment, she had no pain relief.

On her neuropelveologic examination, hyperesthesia and cutaneous allodynia were observed on the right sciatic nerve dermatome. Her muscle strength test and deep tendon reflex test were regular. A vaginal muscle palpation examination was performed in all four quadrants and the Tinel sign was positive on the right sacrospinous ligament. The painful piriformis muscle was palpated at the 8 o'clock position on deep rectal examination. The hip Flexion, Adduction, and Internal Rotation (FAIR) test and forcefully internally rotating the extended thigh on the affected side in the supine position (Frieberg maneuver) were positive on the right side. Extrapelvic etiological causes of piriformis muscle syndrome were excluded with magnetic 
resonance imaging (MRI). The patient was recommended laparoscopic decompression surgery on the right sciatic nerve with a diagnosis of intrapelvic entrapment of the sciatic nerve to relieve sciatica pain.

The second patient was a 30 years old woman with a 2-year history of sciatica pain on her left side. The patient reported her symptoms like shooting and stabbing, and the severity of the pain was $9 / 10$ on the visual analog scale. Her symptoms were continuous and particularly severe when standing position and became unbearable with ten minutes of walking. After the ultrasound-guided injection of local anesthetic into the piriformis muscle, medical, and physical treatment, she had no pain relief.

On her neuropelveologic examination, hyperalgesia and cutaneous allodynia were observed on the left sciatic nerve and left sacral nerve root S2. Her muscle strength test and deep tendon reflex test were regular. A vaginal muscle palpation examination was performed in all four quadrants and the Tinel sign was positive on the left sacrospinous ligament. The hip Flexion, Adduction, and Internal Rotation (FAIR) test and elevation of the flexed leg on the painful side, while the patient is lying on the asymptomatic side (Beatty manoeuver) were positive on the left side. Extrapelvic etiological causes of piriformis muscle syndrome were excluded with magnetic resonance imaging (MRI). With a diagnosis of intrapelvic entrapment of the left sciatic nerve and left sacral nerve root S2, the patient was recommended laparoscopic decompression surgery on the left sciatic nerve and left sacral nerve root $\mathrm{S} 2$ to relieve sciatic pain.

\section{Surgical Procedure}

The first patient underwent standard preparations before the laparoscopic procedures. The patient was placed in the dorsal lithotomy position with a $40^{\circ}$ Trendelenburg position. A 10-mm skin incision was made at the umbilicus level, and the first $10-\mathrm{mm}$ camera port was introduced into the abdominal cavity. Pneumoperitoneum was created with $15-\mathrm{mm} \mathrm{Hg}$ insufflation pressure. A total of four 5-mm lateral ports were placed under direct vision. On laparoscopic exploration, the uterus and ovaries were seen to have been removed.

The adhesions were released and the dissection area was reached. The peritoneal dissection was begun along the right external iliac vessels, and these vessels were separated from the right iliopsoas muscle and the incision expanded and deepened the lumbosacral space by sharp dissection. Meticulous dissection of adjacent tissue surrounding the lumbosacral trunk was performed using a 5-mm LigaSure vessel-sealing device (Blunt Tip; Covidien(r), Dublin, Ireland). Multiple branching superior gluteal vessels in contact with the lumbosacral trunk were coagulated and cut using the LigaSure device. After mobilization of the lymph-fatty tissue from the internal obturator muscle, the sciatic nerve's exposure was obtained. After exposure to the sciatic nerve, the Laparoscopic neuro-navigation (LANN) technique was used to identify the sciatic nerve and sacral roots (5). On further dissection, the pelvic portion of the right sciatic nerve was entrapped by the piriformis muscle bundle was identified (Figure1). The piriformis muscle was gently dissected and transversely separated from the right sciatic nerve and then the muscle was sealed and cut (Figure 2 ); in the end, the right sciatic nerve was wholly released from the piriformis muscle (Figure 3 ). The patient was discharged from the hospital on the second postoperative day without any complications. One day later, just after the operation, her pain decreased dramatically (VAS score 2/10) and her pelvic pain and sciatalgia were entirely resolved. After the 3 and 6 months follow-up, her VAS score decreased to $0 / 10$. The cutaneous allodynia and hyperesthesia along the right sciatic nerve dermatome were entirely resolved.

The second patient also underwent standard preparations before the laparoscopic procedures. The patient was placed in the dorsal lithotomy position with a 40deg Trendelenburg position. A 10-mm skin incision was made at the umbilicus level, and the first 10-mm camera port was introduced into the abdominal cavity. Pneumoperitoneum was created with $15-\mathrm{mm} \mathrm{Hg}$ insufflation pressure. A total of four 5-mm lateral ports was placed under direct vision. On laparoscopic exploration, the uterus and ovaries were seen normal.

The peritoneal dissection was begun along the left external iliac vessels, and these vessels were separated from the left iliopsoas muscle and the incision expanded and deepened the lumbosacral space by sharp dissection. The sacral nerve root S1 was identified, dissection proceeded caudally and sacral nerve root S2 was identified. Laparoscopic neuro-navigation (LANN) technique was used for the identification of sacral 
roots (5). Aberrant piriformis muscle lying over the left sciatic nerve and left sacral nerve root SS was seen (Figure 4 ); after further dissection, the aberrant piriformis muscle was gently dissected, sealed, and cut from the sciatic nerve and sacral nerve root S2 (Figure 5 ). The left sciatic nerve and left sacral nerve root S2 were released entirely from piriformis muscle at the end of the procedure (Figure 6 ). The patient was discharged from the hospital on the third postoperative day without any complications. On the first postoperative day, her pain decreased dramatically (VAS score 3/10) and her left sciatic pain was resolved. After the 3 and 6 months follow-up, her VAS score was $1 / 10$. The hyperalgesia and allodynia along the left sciatic nerve and sacral 2 dermatomes were entirely resolved.

\section{DISCUSSION}

Piriformis syndrome (PS) is a functional entrapment of the sciatic nerve around the piriformis muscle. Most experts agree that piriformis syndrome is a non-discogenic cause of sciatalgia. The diagnosis of piriformis syndrome is often complicated and it is under- and overdiagnosed (7).

Many congenital variations between the sciatic nerve and piriformis muscle exist in the pelvis; the sciatic nerve may divide proximally, the nerve may pass through the piriformis muscle's belly through its tendons or be entrapped between the part of the piriformis muscle and pelvic sidewall (7).

Various causes of entrapment of the sciatic nerve by the piriformis muscle have been reported, but the sciatic nerve's compression by the aberrant piriformis muscle in the pelvis is very rare and diagnosis is often uneasy (7). Pelvic piriformis syndrome is diagnosed clinically. Magnetic resonance neurography (MRN) and EMG have limited accuracy in diagnosing pelvic piriformis syndrome because some patients with a standard magnetic resonance neurography (MRN) or EMG have a persistent complaint of significant pain related to sciatica $(9,10)$.

Surgical treatment of piriformis muscle should be reserved for patients that are refractory to conservative treatment modalities. Traditional open surgical methods to treat piriformis syndrome entails the curvilinear incision around the lateral edge of the gluteus maximus muscle with complete release of the piriformis tendon from its insertion on the posterior femur to expose the sciatic nerve. However, open surgery's undesirable effects are scarring due to large incision, prolonged hospitalization, increased blood loss and recovery time (5).

The endoscopic surgical treatment method's advantages are that it allows for a minimally invasive approach that provides a small incision, shorter hospitalization, decreased loss of blood and morbidity compared to the open procedure. This surgical technique's primary limitation is that it does not allow the diagnosis and treatment of pathologies in the pelvic region (9).

A vast number of malformations of the piriformis muscle have been described in the deep gluteal space that can entrap branches of the sciatic nerve, but piriformis muscle pathologies in the pelvic area have been neglected for a long time. The widespread use and development of laparoscopy have revealed that the piriformis muscle's intrapelvic fibers can also entrap the sacral nerve roots and sciatic nerve in the pelvic area. The laparoscopic approach also allows surgical treatment of the sciatic nerve or sacral nerve roots entrapment by the piriformis muscle in the pelvic area (6).

We reported two rare cases: compression of the sacral nerve root S2 and sciatic nerve between the aberrant piriformis muscle and pelvic sidewall. We performed laparoscopic surgery in the pelvic sidewall area to release the sciatic nerve and sacral nerve root S2 from aberrant piriformis muscle in our two cases. The two patients' pain symptoms dramatically improved. The laparoscopic surgical decompression outcome in our patients was excellent.

Pelvic piriformis muscle syndrome is considered a rare case. However, in our cases, laparoscopic topographic exploration of the pelvic nerves showed that for patients with piriformis syndrome symptoms resistant to medical or endoscopic surgical treatment, the piriformis muscle's release in the pelvic area with using laparoscopic surgery should be recommended to relieve the piriformis syndrome pain. 


\section{Conclusion :}

The diagnosis of piriformis syndrome is obscure and elusive, but a systematic approach is helpful. If a diagnosis is determined correctly and pain is refractory to conservative treatment modalities, laparoscopic exploration of the pelvic nerves and piriformis muscle in the pelvic area and surgical treatment, if necessary, can be a good option, particularly for sciatica pain.

The most critical step in diagnostic workup is the suspicion of the pelvic piriformis muscle syndrome. Laparoscopic pelvic exploration of the sacral and sciatic nerves suspected of being compressed by piriformis muscle is the key to an exact diagnosis and surgical treatment. Laparoscopic pelvic decompression surgery can be safer and have higher success rates in experienced hands.

Funding and Acknowledgements: None

Conflicts of interest: The authors have no conflicts of interest relevant to this article. Financial support and sponsorship: None.

We confirm that this work is original and has not been published elsewhere, nor is it currently under consideration for publication elsewhere, and all authors have contributed significantly and agreed with the content of the manuscript.

The authors have no affiliation with any organization in the subject matter discussed in the manuscript.

This research did not receive any specific grant from funding agencies in the public, commercial, or not-forprofit sectors.

\section{Disclosure:}

None declared.

\section{REFERENCES}

1. Michel $\mathrm{F}$ et al. Piriformis muscle syndrome: diagnostic criteria and treatment of a monocentric series of 250 patients. Ann Phys Rehabil Med . 2013 Jul;56(5): 371-83.

2. Moises Fernandez Hernando 1, Luis Cerezal, Luis Perez-Carro, Faustino Abascal, Ana Canga. Deep gluteal syndrome: anatomy, imaging, and management of sciatic nerve entrapments in the subgluteal space. Skeletal Radiol . 2015 Jul;44(7): 919-34.

3. Shane P Cass. Piriformis syndrome: a cause of nondiscogenic sciatica. Curr Sports Med Rep . 2015 Jan;14(1):41-4.

4. John D Paulson. Abdominal and urogenital diseases can often be the cause of lower back pain and sciatic-like symptoms. Pain Manag. 2012 May;2(3):279-94.

5. Possover M, Rhiem K, Chiantera V. The "Laparoscopic Neuro-Navigation" - LANN: from a functional cartography of the pelvic autonomous neurosystem to a new field of laparoscopic surgery. Minim Invasive Ther Allied Technol . 2004 Dec;13(5):362-7.

6. Han SK, Kim YS, Kim TH, Kang SH. Surgical Treatment of Piriformis Syndrome. Clin Orthop Surg . 2017 Jun;9(2):136-144.

7. Cassidy L, Walters A, Bubb K, Shoja MM, Tubbs RS, Loukas M. Piriformis syndrome: implications of anatomical variations, diagnostic techniques, and treatment options. Surg Radiol Anat . 2012 Aug;34(6):479-86.

8. Dere K, Akbas M, Luleci N. A rare cause of a piriformis syndrome. J Back Musculoskelet Rehabil. $2009 ; 22(1): 55-8$.

9. Fishman LM et al. Piriformis syndrome: diagnosis, treatment, and outcome: a 10-year study. Arch Phys Med Rehabil . 2002 Mar;83(3):295-301.

10. Filler AG et al. Sciatica of nondisc origin and piriformis syndrome: diagnosis by magnetic resonance neurography and interventional magnetic resonance imaging with outcome study of resulting treatment. J Neurosurg Spine. 2005 Feb;2(2):99-115. 
11. Knudsen JS, Mei-Dan O, Brick MJ. Piriformis syndrome and endoscopic sciatic neurolysis. Sports Med Arthrosc Rev 2016 Mar;24(1):e1-7.

12. Hogan E, Vora D, Sherman JH. A minimally invasive surgical approach for the treatment of piriformis syndrome: a case series. Chin Neurosurg J 2020 Mar 30;6:8.

\section{Figure Legend:}

Figure 1. Laparoscopic view (Right Lumbosacral Space). Aberrant piriformis muscle lying over the sciatic nerve.

Figure 2. Laparoscopic view (Right Lumbosacral Space). Laparoscopic cutting of piriformis muscle to release the sciatic nerve.

Figure 3. Laparoscopic view (Right Lumbosacral Space)-Post-decompression images of the cut end of the piriformis muscle and sciatic nerve.

Figure 4. Laparoscopic view (Left Lumbosacral Space). Aberrant piriformis muscle lying over the sciatic nerve and S2 nerve root.

Figure 5. Laparoscopic view (Left Lumbosacral Space). Laparoscopic cutting of piriformis muscle to release the sciatic nerve and S2 nerve root.

Figure 6. Laparoscopic view (Left Lumbosacral Space). Post-decompression images of the cut end of the piriformis muscle, sciatic nerve and the $\mathrm{S} 2$ nerve root.

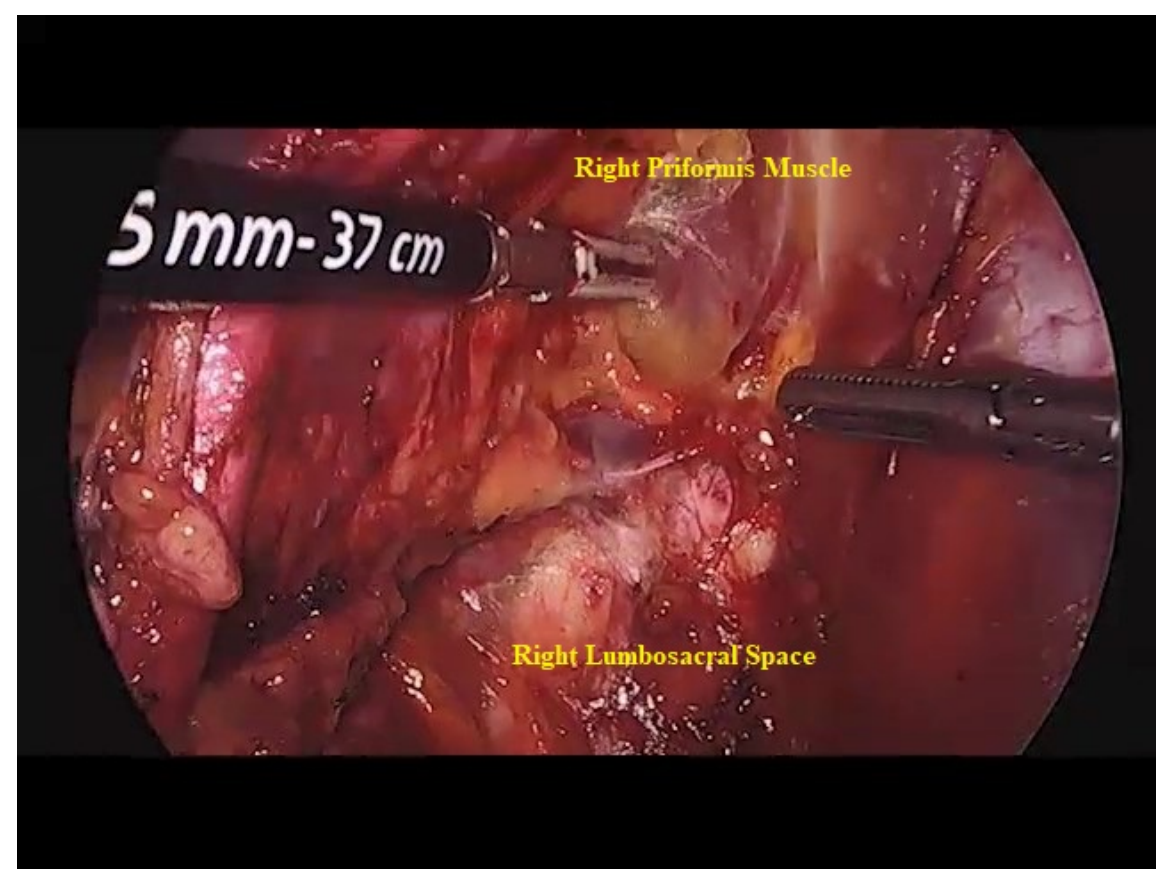



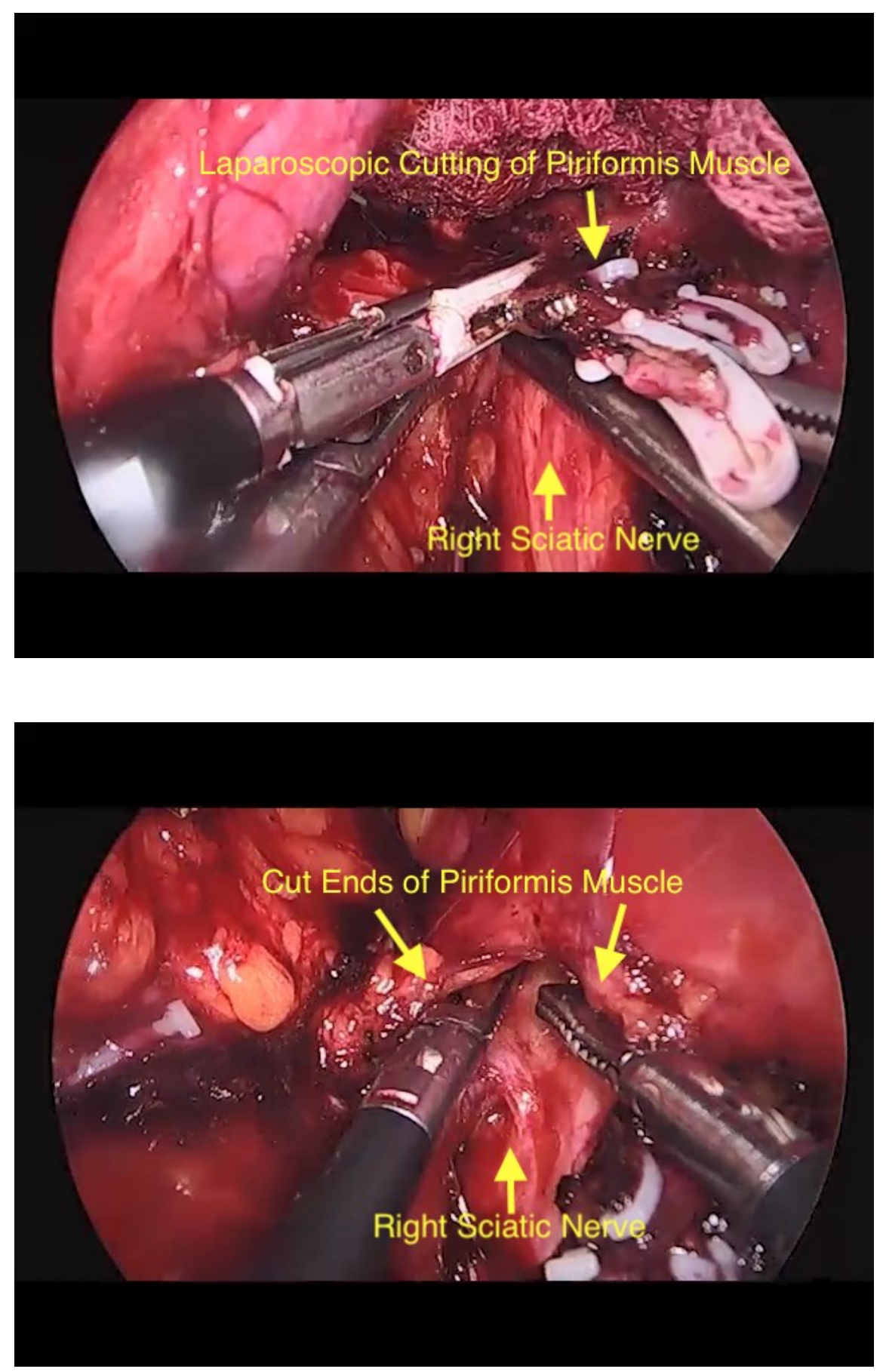

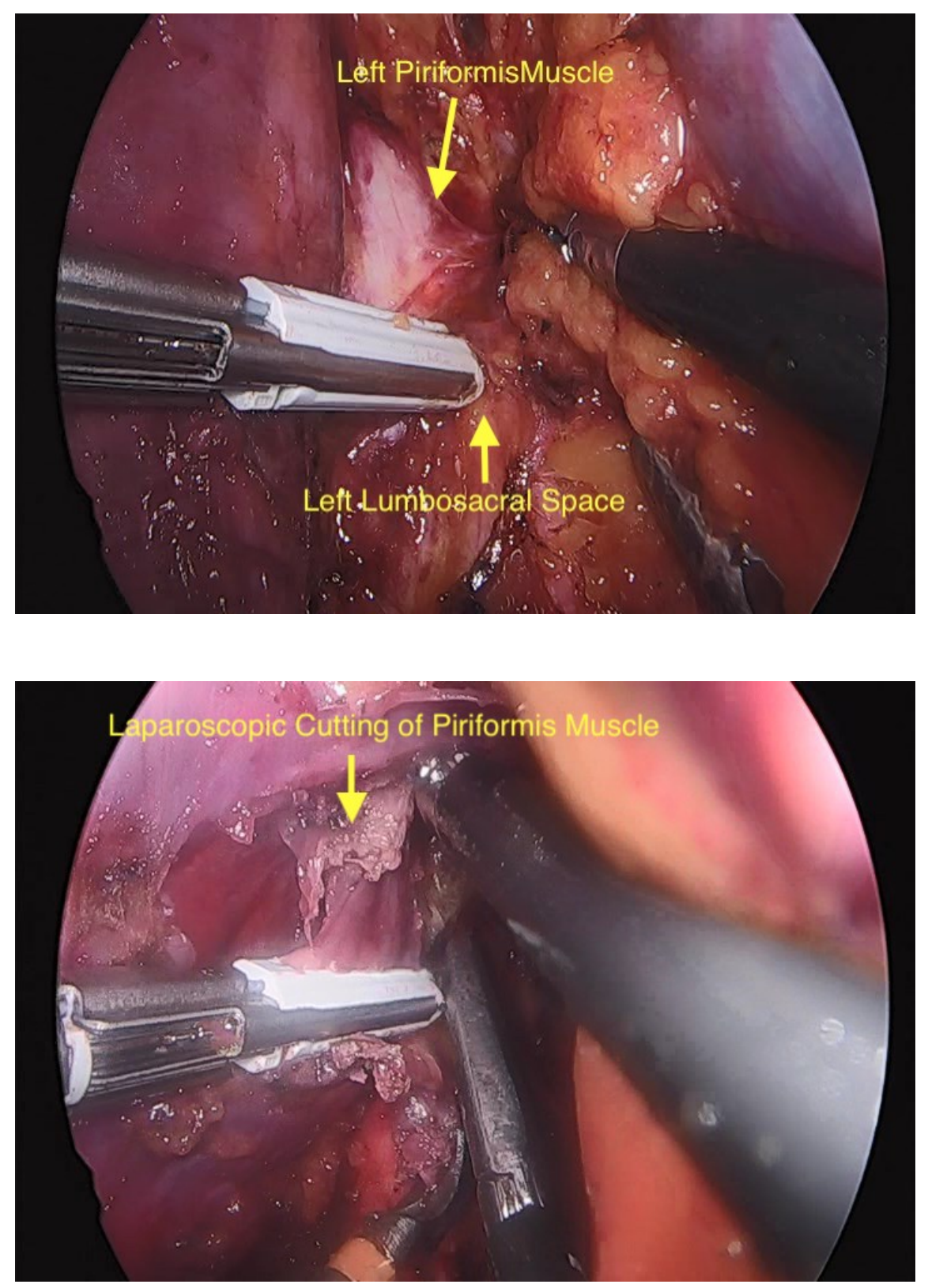


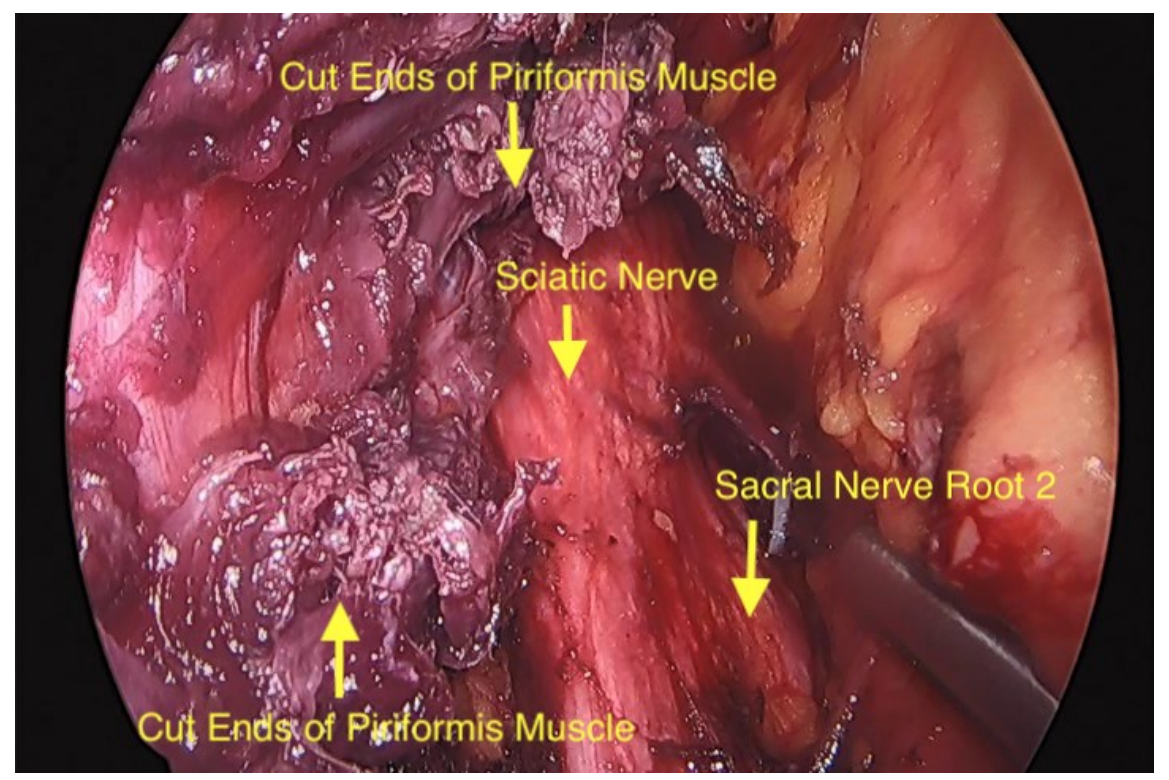

